\title{
Mito-mice $\Delta$ and mitochondrial DNA mutator mice as models of human osteoporosis caused not by aging but by hyperparathyroidism
}

\author{
Takayuki MITO ${ }^{1,2) *}$, Haruna TANI ${ }^{3) *}$, Michiko SUZUKI ${ }^{3)}$, Kaori ISHIKAWA ${ }^{1,3)}$, \\ Kazuto NAKADA ${ }^{1,3)}$, and Jun-Ichi HAYASHI ${ }^{4}$ \\ 1) Faculty of Life and Environmental Sciences, University of Tsukuba, 1-1-1 Tennodai, Tsukuba, Ibaraki 305-8572, \\ Japan \\ 2) Japan Society for the Promotion of Science (JSPS), 8 Ichiban-cho, Chiyoda-ku, Tokyo 102-8472, Japan \\ ${ }^{3)}$ Graduate School of Life and Environmental Sciences, University of Tsukuba, 1-1-1 Tennodai, Tsukuba, Ibaraki \\ 305-8572, Japan \\ 4) University of Tsukuba, 1-1-1 Tennodai, Tsukuba, Ibaraki 305-8572, Japan
}

\begin{abstract}
Mitochondrial DNA (mtDNA) mutator mice showing accelerated accumulation of mtDNA with somatic mutations are potentially useful models of human aging, whereas mito-mice $\Delta$ showing accelerated accumulation of mtDNA with a deletion mutation ( $\triangle \mathrm{mtDNA})$ are potentially useful models of mitochondrial diseases but not human aging, even though both models express an age-associated decrease in mitochondrial respiration. Because osteoporosis is the only premature aging phenotype observed in mtDNA mutator mice with the C57BL/6J nuclear genetic background, our previous study precisely examined its expression spectra and reported that both mtDNA mutator mice and mitomice $\Delta$, but not aged mice, developed decreased cortical bone thickness. Moreover, decreased cortical bone thickness is usually not seen in aged humans but is commonly seen in the patients with hyperparathyroidism caused by oversecretion of parathyroid hormone (PTH). In the present study, we showed higher concentrations of blood PTH in mtDNA mutator mice and mito-mice $\Delta$ than in aged mice. We also found that both models developed decreased mitochondrial respiration in the duodenum or renal tubules, which would lead to hypocalcemia, oversecretion of PTH, and ultimately osteoporosis. Thus, mtDNA mutator mice and mito-mice $\Delta$ may be useful models of human osteoporosis caused not by aging but by hyperparathyroidism.
\end{abstract}

Key words: human aging, hyperparathyroidism, mito-mice $\Delta$, mtDNA mutator mice, osteoporosis

\section{Introduction}

The accumulation of mitochondrial DNA (mtDNA) with pathogenic mutations that induce defective mitochondrial respiration is a possible underlying cause of the development of mitochondrial diseases [25]. Previously, we generated mito-mice $\Delta$ (mice harbouring mtDNA with a large-scale deletion $[\Delta \mathrm{mtDNA}])$ : the deletion was 4,696 bp long with a breaking point from nucleotide position 7,759 in the $m t$ - $T k$ gene to 12,454 in

(Received 25 April 2018 / Accepted 6 June 2018 / Published online in J-STAGE 4 July 2018)

*These authors contributed equally to this work.

Address corresponding: J.-I. Hayashi, University of Tsukuba, 1-1-1 Tennodai, Tsukuba, Ibaraki 305-8572, Japan

Supplementary Figure: refer to J-STAGE: https://www.jstage.jst.go.jp/browse/expanim

(c) $(\$)$ This is an open-access article distributed under the terms of the Creative Commons Attribution Non-Commercial No Derivatives (by-nc-nd) License <http://creativecommons.org/licenses/by-nc-nd/4.0/> 
the $m t-N d 5$ gene, and it included 6 tRNA genes and 7 structural genes [11]. Then, we showed that these mice developed phenotypes of mitochondrial diseases only when $\triangle \mathrm{mtDNA}$ had accumulated predominantly to reduce mitochondrial respiratory function $[11,19]$. These results provided direct evidence indicating that mitochondrial diseases are the result of defective mitochondrial respiration caused by the accumulation of mtDNA with a pathogenic mutation.

Because small amounts of mtDNA with the same pathogenic mutations as those found in patients with mitochondrial diseases accumulate in the tissues of normal elderly humans, the mitochondrial theory of aging $[13,14,23,25]$ proposes that accumulation of mtDNA with pathogenic mutations is responsible not only for the development of mitochondrial diseases but also for the development of human aging and age-associated disorders. This hypothesis is supported by mtDNA mutator mice, which are generated by the introduction of a proofreading-deficient mtDNA polymerase gene $[2,16$, 24]; during mtDNA replication in these mice, defective proofreading results in accelerated accumulation of somatic mutations in their mtDNA with aging and simultaneous development of early onset defective mitochondrial respiration and premature aging phenotypes.

In contrast, mito-mice $\Delta$ show accelerated accumulation of $\triangle \mathrm{mtDNA}$ with age due to its smaller size compared with wild-type mtDNA and simultaneously express early onset defective mitochondrial respiration and phenotypes related to mitochondrial diseases but do not express premature aging phenotypes [11, 19]. So why premature aging phenotypes are observed in mtDNA mutator mice but not in mito-mice $\Delta$ even though both model mice develop age-associated defective mitochondrial respiration is an issue that has not yet been resolved.

Previously [18], we reported that one possible reason behind the exclusive development of premature aging phenotypes in mtDNA mutator mice could be the differences in the nuclear genetic background used to generate these model mice. That is, when mtDNA mutator mice shared the same nuclear genetic background as mitomice $\Delta$, i.e., C57BL/6J (hereafter referred to as B6J), both models developed kyphosis but not alopecia or hair graying [18], which have been reported to develop in mtDNA mutator mice as premature aging phenotypes $[16,24]$. Indeed, differences in nuclear genetic background have also been shown to affect phenotypic spectrum even in inbred mice $[5,6,8,15]$.
Because most human kyphosis is caused by osteoporosis [20], previously we examined trabecular and cortical bone thickness and found decreased cortical bone thickness in mito-mice $\Delta$ and mtDNA mutator mice but not in aged mice [17]. Moreover, human age-associated osteoporosis is frequently characterized by decreased trabecular bone thickness without decreased cortical bone thickness [20]. This suggests that decreased cortical bone thickness is not associated with aging in either mice or humans. In contrast, decreased cortical bone thickness together with osteoporosis, which we reported in mito-mice $\Delta$ and mtDNA mutator mice [17], is seen in patients with hyperparathyroidism, which is caused by excessive secretion of parathyroid hormone (PTH) from the parathyroid glands in response to hypocalcaemia [4].

Therefore, this study examined whether mito-mice $\Delta$ and mtDNA mutator mice can be used as models of human osteoporosis caused by aging or hyperparathyroidism.

\section{Materials and Methods}

\section{Mice}

Inbred B6J male mice generated by sibling mating more than 40 times were obtained from CLEA Japan (Tokyo, Japan). Mito-mice $\Delta$ [11] and mtDNA mutator mice [18] sharing the B6J nuclear genetic background were generated as reported previously. We used 10 -month-old mito-mice $\Delta$ with $81.2 \pm 1.3 \% \Delta$ mtDNA in their tails and 10-month-old mtDNA mutator mice homozygous for the mtDNA polymerase editing mutation, PolgD257A, because these mice had osteoporosis [17] corresponding to a phenotype prevalently observed in elderly human subjects. The proportion of $\triangle \mathrm{mtDNA}$ in their tissues was deduced by its estimation using the tails, because it did not differ significantly among different tissues from the same mito-mice $\Delta$ [11]. The proportion of $\triangle \mathrm{mtDNA}$ in their tails was determined by means of real-time polymerase chain reaction analysis, as described previously [21]. With respect to the mutations in mtDNA mutator mice, sequence analysis revealed that 22 point mutations were accumulated in fifty clones of $517 \mathrm{bp}$-fragments of the $m t$-Col gene in the mtDNA prepared from the heart of an mtDNA mutator mouse (10 months old), while no mutations were found in the heart of an age-matched B6J mouse [18]. We also used 10-month-old B6J mice and 27-month-old B6J mice as age-matched controls and aged mice, respectively. Ani- 
mal experiments were performed in accordance with protocols approved by the Experimental Animal Committee of the University of Tsukuba, Japan.

\section{Measurement of serum PTH and $\mathrm{Ca}^{2+}$ concentrations}

Serum PTH concentrations were measured by using a mouse PTH 1-84 ELISA Kit (Immutopics, Inc., Athens, $\mathrm{OH}, \mathrm{USA}$ ). Serum $\mathrm{Ca}^{2+}$ concentrations were measured by using the o-cresolphthalein complexone method (Nescoat Ca-V2, Alfresa, Tokyo, Japan).

\section{Histological analyses}

Paraffin-embedded sections of decalcified femurs were subjected to tartrate-resistant acid phosphatase (TRAP) staining by using a TRAP/ALP staining kit (Wako Pure Chemical Industries, Osaka, Japan). The percentage of bone surface covered with osteoclasts was calculated from TRAP-stained sections by using the Leica Application Suite V4.5 software (Leica, Wetzlar, Hesse, Germany). Histochemical analyses for COX and succinate dehydrogenase activities were performed as described previously [18] by using cryosections (thickness, $10 \mu \mathrm{m}$ ) of renal or duodenal tissue. Haematoxylin and eosinstained sections were used for histopathological analysis of renal tissue.

\section{Measurement of blood urea nitrogen (BUN)}

Blood samples were collected via the retro-orbital vein. BUN concentrations were determined by using a Urea NB test kit (Wako Pure Chemical Industries).

\section{Statistical analysis}

Data were analysed by using Dunnett's test or one-way ANOVA followed by Dunnett's post hoc test. $P<0.05$ was considered statistically significant.

\section{Results}

First, we determined the serum concentrations of PTH, and we found a significantly higher serum PTH concentration both in mito-mice $\Delta$ and mtDNA mutator mice (10 months old) compared with age-matched control B6J mice (10 months old; $P<0.05)$ or aged B6J mice (27 months old; $P<0.05$ ) (Fig. 1).

Excessive secretion of PTH enhances bone resorption via osteoclast activation, resulting in decreased cortical bone thickness and osteoporosis [4]. Therefore, next we estimated the percentage of cortical bone and trabecular

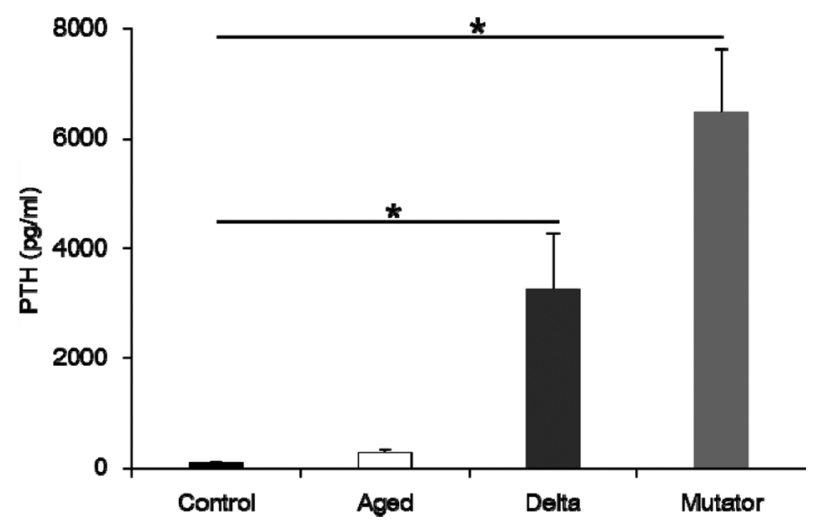

Fig. 1. Comparison of serum parathyroid hormone (PTH) levels between age-matched control mice (Control; 10-month-old mice as age-matched controls), aged mice (Aged; 27-month-old mice), mito-mice $\Delta$ (Delta; 10 -month-old mito-mice $\Delta$ ), and mtDNA mutator mice (Mutator; 10-month-old mtDNA mutator mice). The amount of $\triangle$ mtDNA in the tail of the mito-mice $\Delta$ was $81.2 \pm 1.3 \%$. Data are presented as the mean $+\mathrm{SD} .{ }^{*} P<0.05(\mathrm{n}=3)$.

bone covered by osteoclasts in histological sections of femur. In mito-mice $\Delta$ and mtDNA mutator mice, the percentage of the surface of cortical bone (Fig. 2A), but not trabecular bone (Fig. 2B), covered by osteoclasts was significantly larger than that of age-matched control B6J mice (10 months old; $P<0.05$ ), suggesting that mitomice $\Delta$ and mtDNA mutator mice develop pathological features that are similar to those seen in human hyperparathyroidism [4]. In addition, significantly less cortical bone (Fig. 2A) and trabecular bone (Fig. 2B) surface was covered by osteoclasts in aged 27-month-old B6J mice than in 10-month-old control B6J mice $(P<0.05)$, which is consistent with previous observations reported in aged humans [1].

Patients with hyperparathyroidism also have elevated serum concentrations of circulating ionized calcium $\left(\mathrm{Ca}^{2+}\right)$ (i.e., hypercalcaemia) due to increased cortical bone resorption [4]. Therefore, we determined the serum concentrations of $\mathrm{Ca}^{2+}$ and found significantly higher concentrations of $\mathrm{Ca}^{2+}$ in mito-mice $\Delta$ and mtDNA mutator mice but not in aged $\mathrm{B} 6 \mathrm{~J}$ mice compared with control B6J mice $(P<0.05$; Fig. 3$)$.

Together, these observations suggest that in mitomice $\Delta$ and mtDNA mutator mice, increased serum concentrations of PTH (Fig. 1) causes increased recruitment of osteoclasts to the surface of cortical bone (Fig. 2A), which results in increased bone resorption leading to increased serum concentrations of $\mathrm{Ca}^{2+}$ (hypercalcaemia; 
A
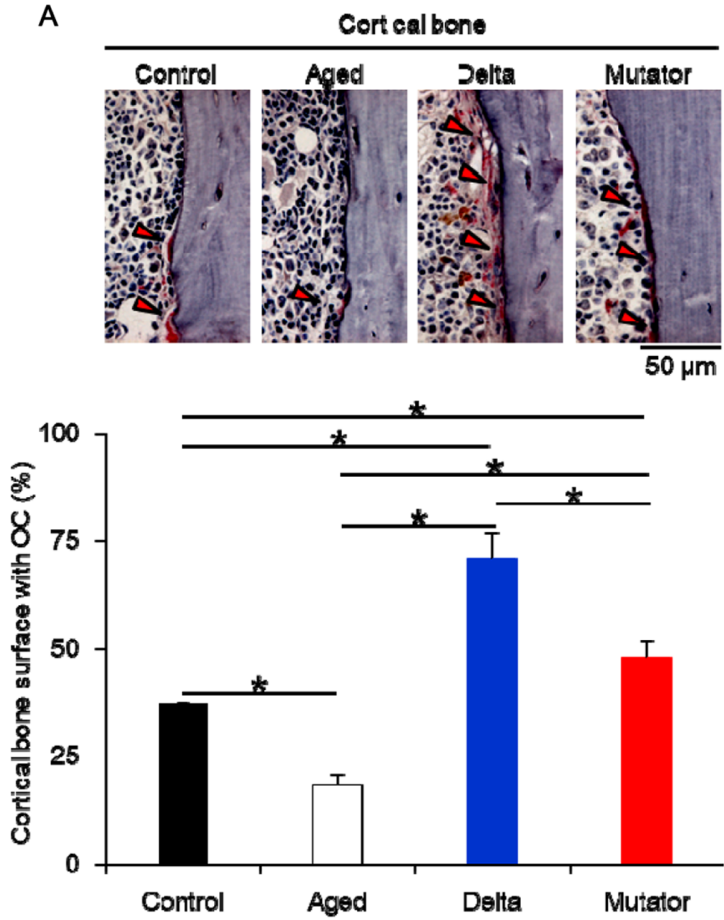

B
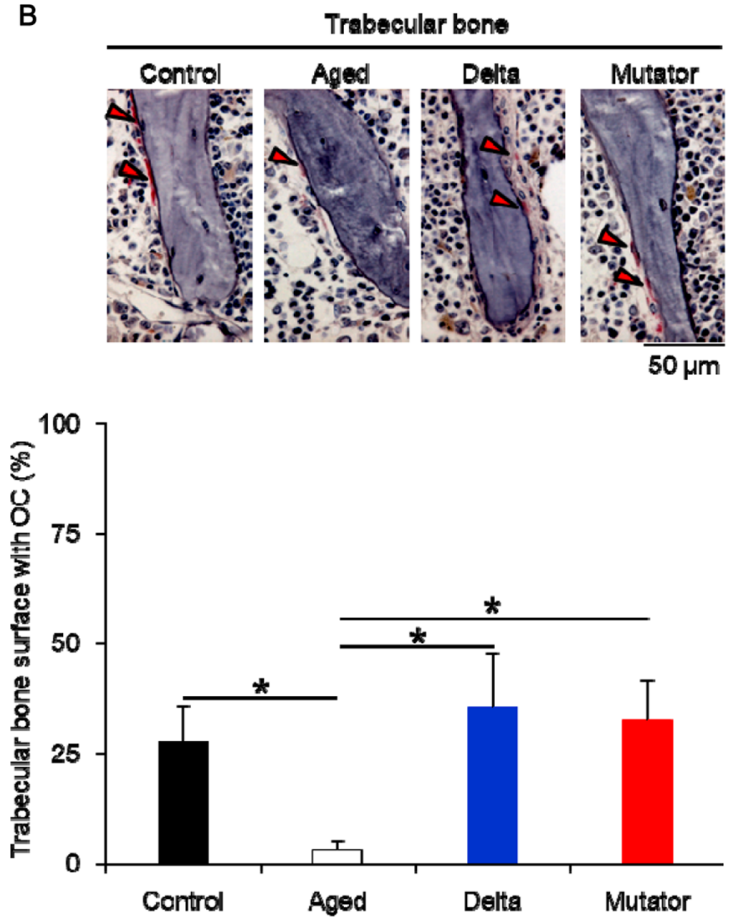

Fig. 2. Histopathological analysis of osteoclast coverage of the surface of cortical bone (A) and trabecular bone (B) after tartrate-resistant acid phosphatase (TRAP) staining in age-matched control mice (Control; 10-month-old mice as age-matched controls), aged mice (Aged; 27-month-old mice), mito-mice $\Delta$ (Delta; 10 -month-old mito-mice $\Delta$ ), and mtDNA mutator mice (Mutator; 10-month-old mtDNA mutator mice). The amount of $\triangle$ mtDNA in the tail of the mito-mice $\Delta$ was $81.2 \pm 1.3 \%$. Upper panels: Histological analysis. Red arrowheads indicate osteoclasts (OCs) stained red with TRAP. Lower panels: Percentage of bone surface covered with OCs. The percentage of the bone surface covered with OCs was determined from the histological sections shown in the upper panels by using the Leica Application Suite V4.5 software (Leica, Wetzlar, Hesse, Germany). Data are presented as the mean + SD. $* P<0.05$ $(\mathrm{n}=3)$. Scale bars, $50 \mu \mathrm{m}$.

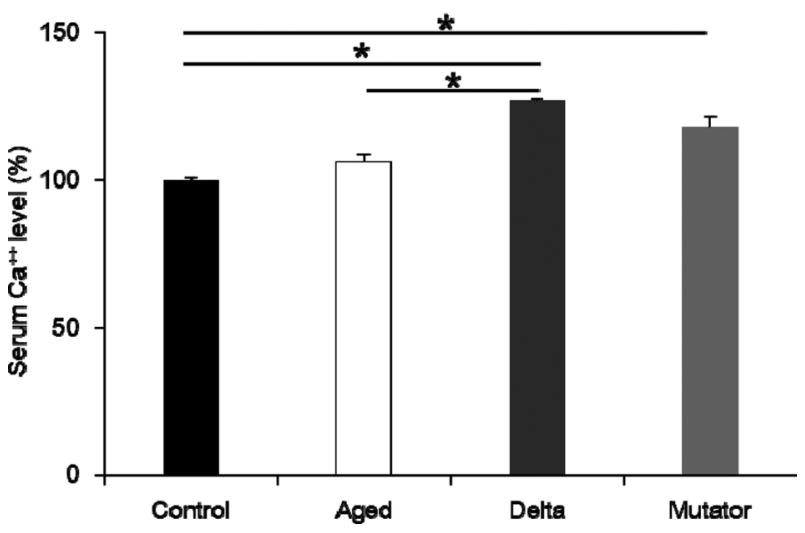

Fig. 3. Serum circulating ionized calcium $\left(\mathrm{Ca}^{2+}\right)$ levels in agematched control mice (Control; 10-month-old mice as agematched controls), aged mice (Aged; 27-month-old mice), mito-mice $\Delta$ (Delta; 10 -month-old mito-mice $\Delta$ ), and $\mathrm{mtD}$ NA mutator mice (Mutator; 10-month-old mtDNA mutator mice). The amount of $\triangle \mathrm{mtDNA}$ in the tail of the mitomice $\Delta$ was $81.2 \pm 1.3 \%$. Data are presented as the mean + SD. $* P<0.05(\mathrm{n}=3)$.
Fig. 3) and decreased cortical bone thickness (osteoporosis) [17].

Next, we examined why the serum concentration of PTH was higher in mito-mice $\Delta$ and mtDNA mutator mice than in age-matched control mice and aged mice (Fig. 1). In patients with hyperparathyroidism, impaired renal tubular reabsorption of $\mathrm{Ca}^{2+}$ from urine or impaired duodenal absorption of $\mathrm{Ca}^{2+}$ from food leads to hypocalcaemia and increased secretion of PTH in response to hypocalcaemia [4]. Thus, we hypothesized that defective mitochondrial respiration in the kidney and duodenum in mito-mice $\Delta$ and mtDNA mutator mice, respectively, causes hypocalcaemia leading to increased secretion of PTH.

Anaemia and a granulated surface of the kidney were exclusively observed in mito-mice $\Delta$ (Fig. 4A, upper). Then, we conducted a histochemical analysis of mitochondrial respiration in the kidneys of mito-mice $\Delta$ and mtDNA mutator mice and found that a significant reduc- 
A
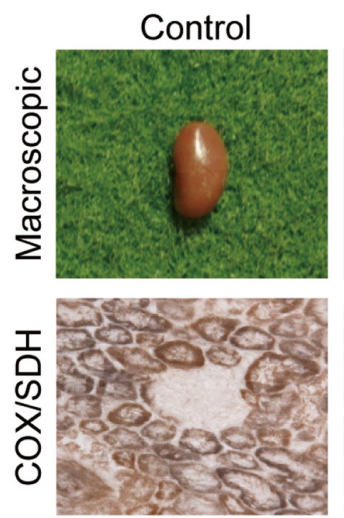

岌

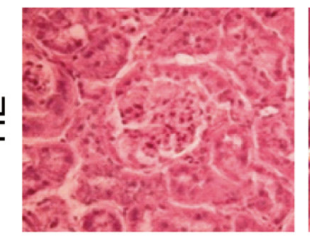

Aged
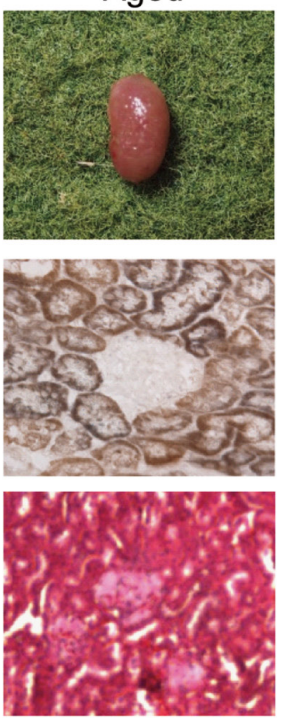

Delta
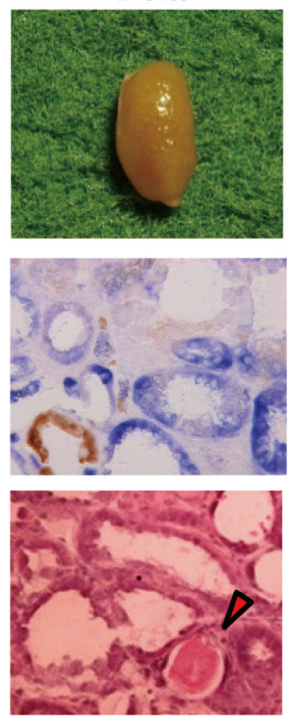

Mutator
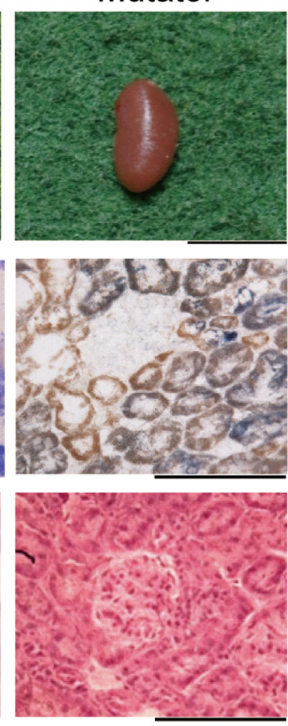

*

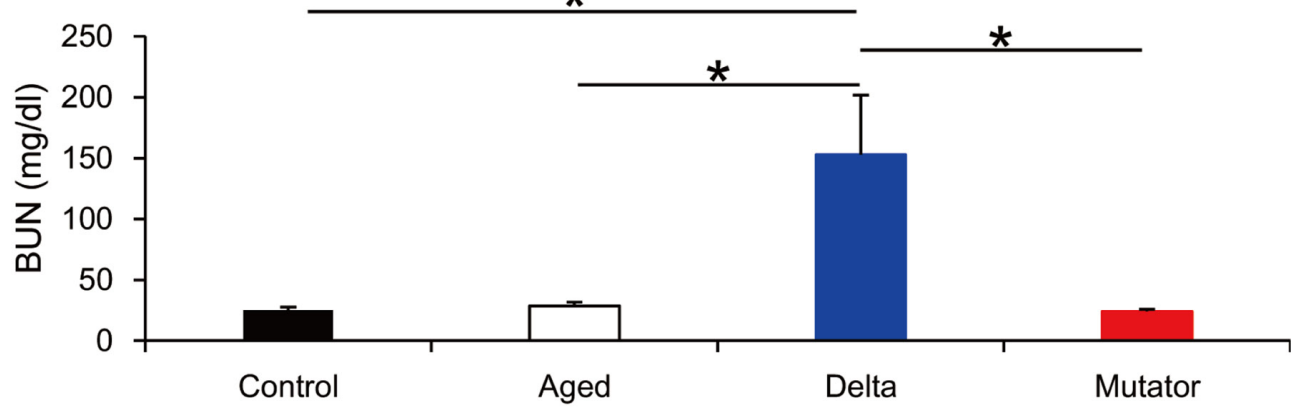

B

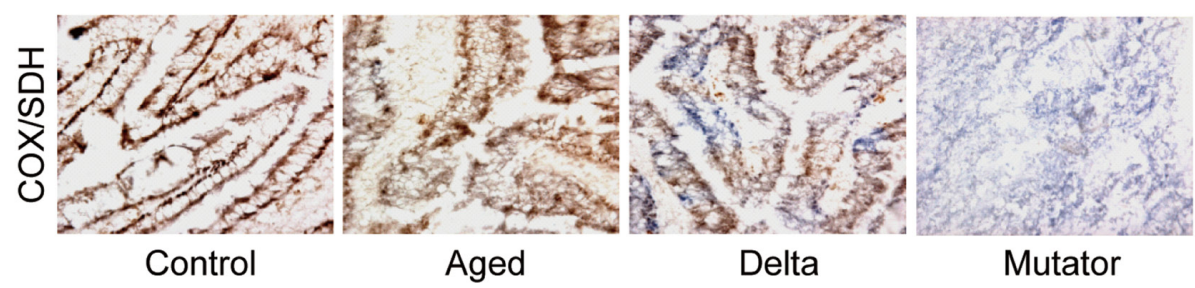

Fig. 4. Macroscopic and histopathological analyses of the kidney and duodenum of age-matched control mice (Control; 10-month-old mice as age-matched controls), aged mice (Aged; 27-month-old mice), mito-mice $\Delta$ (Delta; 10-month-old mito-mice $\Delta$ ), or mtDNA mutator mice (Mutator; 10-month-old mtDNA mutator mice). The amount of $\Delta$ mtDNA in the tail of the mito-mice $\Delta$ was $81.2 \pm 1.3 \%$. (A) Abnormalities of the kidney. Macroscopy: Macroscopic view showing anaemia and the granulated surface of a representative mito-mice $\Delta$ kidney. Scale bar, $1 \mathrm{~cm}$. COX/SDH: Tissue sections were stained for cytochrome c oxidase (COX) and succinate dehydrogenase (SDH). COX and SDH activities were detected as a brown and blue colours, respectively. Because mtDNA encodes three subunits of COX and no subunits of SDH, mutations in mtDNA reduced COX but not SDH activity. Therefore, tissues with reduced COX activity appear to contain more blue regions. COX/SDH staining revealed the presence of defective mitochondrial respiration in mito-mice $\Delta$ and mtDNA mutator mice but not in aged mice. Scale bar, $100 \mu \mathrm{m}$. HE: Haematoxylin and eosin (HE) staining revealed the presence of renal tubules with dilated lumens and casts (arrowhead) in mito-mice $\Delta$. Scale bar, $100 \mu \mathrm{m}$. Increased blood urea nitrogen (BUN) levels were also found in mito-mice $\Delta$. Data are presented as the mean $+\mathrm{SD}$. ${ }^{*} P<0.05(\mathrm{n}=3)$. (B) Abnormalities of duodenum. COX/SDH staining revealed defective mitochondrial respiration in mito-mice $\Delta$ and $m t D N A$ mutator mice but not in aged mice. 
tion in cytochrome c oxidase (COX) activity was observed in mito-mice $\Delta$ (Fig. $4 \mathrm{~A}$, middle). A histopathological analysis of the kidney also revealed renal tubules with dilated lumens and casts in mito-mice $\Delta$ (Fig. 4A, lower). Moreover, a significantly higher serum concentration of BUN $(P<0.05)$, which is a marker of renal failure, was observed in mito-mice $\Delta$ compared with mtDNA mutator mice, age-matched control mice, and aged mice $(P<0.05$; Fig. 4A, graph). The development of renal failure in mito-mice $\Delta$ but not in mtDNA mutator mice (Fig. 4A) is consistent with our previous observations [18], although the reasons why renal failure exclusively develops in mito-mice $\Delta$ remain unknown.

It has also been suggested that mtDNA mutator mice develop markedly defective mitochondrial respiration in the duodenum [3]. Our histochemical analysis also revealed a significant reduction in COX activity in the duodenum of mtDNA mutator mice (Fig. 4B). The absence of an age-associated decline in mitochondrial respiration in aged mice with the B6J nuclear background (Fig. 4) is consistent with the results of our previous study [22].

It is likely that the preferential expression of the respiration defects in the kidney of mito-mice $\Delta$ and in the duodenum of mtDNA mutator mice are due partly to a slightly higher accumulation of $\triangle \mathrm{mtDNA}$ in the kidney of mito-mice $\Delta[11]$ and a slightly higher accumulation of somatic mtDNA mutations in the duodenum of mtDNA mutator mice [16]. Because renal tubules and the duodenum are important for $\mathrm{Ca}^{2+}$ reabsorption and $\mathrm{Ca}^{2+}$ absorption, respectively, defective mitochondrial respiration in the kidney and/or duodenum (Fig. 4) would result in reduced serum concentrations of $\mathrm{Ca}^{2+}$ (hypocalcaemia). However, we are unable to explain the exact mechanisms of how respiration defects induce impaired $\mathrm{Ca}^{2+}$ reabsorption in the kidney and impaired $\mathrm{Ca}^{2+}$ absorption in the duodenum. Hypocalcaemia causes increased secretion of $\mathrm{PTH}$, and the resultant high serum concentration of PTH (Fig. 1) induces bone reabsorption to compensate for the hypocalcaemia via activating osteoclasts (Fig. 2A), leading to hypercalcaemia (Fig. 3), decreased cortical bone thickness [17], and osteoporosis [4].

Considering that the aged mice developed neither defective mitochondrial respiration (Fig. 4) nor increased serum concentrations of PTH (Fig. 1), we conclude that osteoporosis in mito-mice $\Delta$ and mtDNA mutator mice develops via respiration defects and hyperparathyroidism but not via aging (Supplementary Fig. S1).

\section{Discussion}

In the present study, we precisely examined the development of osteoporosis, one of the premature aging phenotypes observed in mtDNA mutator mice [16, 24], for three reasons. First, age-associated kyphosis caused by osteoporosis is the only premature aging phenotype observed in mtDNA mutator mice with the B6J nuclear genetic background [18]. Second, development of osteoporosis due to decreased cortical bone thickness has been shown in mito-mice $\Delta$ and mtDNA mutator mice [17] but not in aged mice [17] or aged humans [20]. Third, decreased cortical bone thickness has been reported in patients with hyperparathyroidism [4].

To examine whether or not mito-mice $\Delta$ and mtDNA mutator mice could be useful models of human osteoporosis associated with hyperparathyroidism rather than human aging, we determined the serum concentrations of PTH and found higher concentrations in mito-mice $\Delta$ and mtDNA mutator mice than in aged mice (Fig. 1). In addition, a histopathological analysis revealed reduced mitochondrial respiration in the kidney (Fig. 4A) and duodenum (Fig. 4B) in mito-mice $\Delta$ and mtDNA mutator mice. This suggests that impaired $\mathrm{Ca}^{2+}$ reabsorption from urine via the renal tubules or impaired $\mathrm{Ca}^{2+}$ absorption from food via the duodenum due to defective mitochondrial respiration leads to hypocalcaemia, which in turn stimulates excessive secretion of PTH from the parathyroid glands (Supplementary Fig. S1).

We also found that mito-mice $\Delta$ and mtDNA mutator mice not only have defective mitochondrial respiration and high serum concentrations of PTH but also increased recruitment of osteoclasts to the cortical bone surface (Fig. 2A), which results in decreased cortical bone thickness and hypercalcaemia (Fig. 3). Although decreased cortical bone thickness was not observed in aged mice [17] and in aged human subjects [20], this phenotype has been reported in humans with hyperparathyroidism [4]. This suggests that mito-mice $\Delta$ and mtDNA mutator mice may be useful models for investigating the pathogenesis of the osteoporosis that develops in humans in association with hyperparathyroidism rather than age (Supplementary Fig. S1).

The mitochondrial theory of aging proposes that human aging is caused by accumulation of somatic mutations in mtDNA leading to defective mitochondrial respiration [13, 14, 23, 25]. However, we recently reported that the age-associated respiration defects in elderly 
human fibroblasts could be restored by reprogramming them (isolation of induced pluripotent stem cells [iPSCs] from fibroblast and redifferentiation of them into fibroblasts) $[7,10]$. Therefore we proposed an alternative theory that human fibroblasts isolated from elderly subjects develop defective mitochondrial respiration not due to mutations in their mtDNA $[7,9,12]$ but due to epigenetic downregulation of nuclear-encoded genes [7, 10].

In contrast, the mitochondrial theory of aging is supported by the creation of mtDNA mutator mice because of their increased accumulation of somatic mutations in mtDNA and consequent development of defective mitochondrial respiration and premature aging phenotypes $[2,16,24]$. However, the present study revealed that the mechanism underlying the development of osteoporosis, which corresponds to the only premature aging phenotype observed in mtDNA mutator mice with the B6J nuclear genetic background [18], is fundamentally different from the mechanism underlying the development of osteoporosis in aged mice or aged humans. Therefore, the phenotypes observed in mtDNA mutator mice do not necessarily support the concept that accumulation of mtDNA with pathogenic mutations is responsible for the development of aging phenotypes.

\section{Author contributions}

T. M. and J. -I. H. conceived and designed the experiments. T. M., H. T., and M. S. conducted the overall experiments. K. I. and K. N. helped with the design and coordination of the study. T. M., H. T., and J. -I. H. wrote the paper. All authors reviewed the manuscript.

\section{Conflict of Interests}

The authors declare that there are no competing financial interests.

\section{Acknowledgments}

This work was supported by Grants-in-Aid for Scientific Research A (25250011 to J. -I. H.), Scientific Research B (16H0478 to K. N.), Challenging Exploratory Research (16K14719 to K. N.), and Young Scientists B (16K18535 to K. I.) from the Japan Society for the Promotion of Science, and by Life Sciences Fellowships from Takeda Science Foundation to K.I. This work was also supported by a Grant-in-Aid for Japan Society for the Promotion of Science Fellows (No. 26-467) to T. M.

\section{References}

1. Boskey, A.L. and Coleman, R. 2010. Aging and bone. $J$. Dent. Res. 89: 1333-1348. [Medline] [CrossRef]

2. Bratic, A. and Larsson, N.G. 2013. The role of mitochondria in aging. J. Clin. Invest. 123: 951-957. [Medline] [CrossRef]

3. Fox, R.G., Magness, S., Kujoth, G.C., Prolla, T.A., and Maeda, N. 2012. Mitochondrial DNA polymerase editing mutation, PolgD257A, disturbs stem-progenitor cell cycling in the small intestine and restricts excess fat absorption. Am. J. Physiol. Gastrointest. Liver Physiol. 302: G914-G924. [Medline] [CrossRef]

4. Fraser, W.D. 2009. Hyperparathyroidism. Lancet 374: 145158. [Medline] [CrossRef]

5. Freeman, D., Lesche, R., Kertesz, N., Wang, S., Li, G., Gao, J., Groszer, M., Martinez-Diaz, H., Rozengurt, N., Thomas, G., Liu, X., and Wu, H. 2006. Genetic background controls tumor development in PTEN-deficient mice. Cancer Res. 66: 6492-6496. [Medline] [CrossRef]

6. Hashizume, O., Yamanashi, H., Taketo, M.M., Nakada, K., and Hayashi, J.I. 2015. A specific nuclear DNA background is required for high frequency lymphoma development in transmitochondrial mice with G13997A mtDNA. PLoS One 10: e0118561. [Medline] [CrossRef]

7. Hashizume, O., Ohnishi, S., Mito, T., Shimizu, A., Ishikawa, K., Nakada, K., Soda, M., Mano, H., Togayachi, S., Miyoshi, H., Okita, K., and Hayashi, J.I. 2015. Epigenetic regulation of the nuclear-coded GCAT and SHMT2 genes confers human age-associated mitochondrial respiration defects. Sci. Rep. 5: 10434. [Medline] [CrossRef]

8. Hashizume, O., Shimizu, A., Yokota, M., Sugiyama, A., Nakada, K., Miyoshi, H., Itami, M., Ohira, M., Nagase, H., Takenaga, K., and Hayashi, J.I. 2012. Specific mitochondrial DNA mutation in mice regulates diabetes and lymphoma development. Proc. Natl. Acad. Sci. USA 109: 10528-10533. [Medline] [CrossRef]

9. Hayashi, J.I., Ohta, S., Kagawa, Y., Kondo, H., Kaneda, H., Yonekawa, H., Takai, D., and Miyabayashi, S. 1994. Nuclear but not mitochondrial genome involvement in human agerelated mitochondrial dysfunction. Functional integrity of mitochondrial DNA from aged subjects. J. Biol. Chem. 269: 6878-6883. [Medline]

10. Hayashi, J.I., Hashizume, O., Ishikawa, K., and Shimizu, A. 2016. Mutations in mitochondrial DNA regulate mitochondrial diseases and metastasis but do not regulate aging. Curr. Opin. Genet. Dev. 38: 63-67. [Medline] [CrossRef]

11. Inoue, K., Nakada, K., Ogura, A., Isobe, K., Goto, Y., Nonaka, I., and Hayashi, J.I. 2000. Generation of mice with mitochondrial dysfunction by introducing mouse mtDNA carrying a deletion into zygotes. Nat. Genet. 26: 176-181. [Medline] [CrossRef]

12. Isobe, K., Ito, S., Hosaka, H., Iwamura, Y., Kondo, H., Kagawa, Y., and Hayashi, J.I. 1998. Nuclear-recessive mutations of factors involved in mitochondrial translation are responsible for age-related respiration deficiency of human skin fibroblasts. J. Biol. Chem. 273: 4601-4606. [Medline] [CrossRef] 
13. Jacobs, H.T. 2003. The mitochondrial theory of aging: dead or alive? Aging Cell 2: 11-17. [Medline] [CrossRef]

14. Khrapko, K. and Vijg, J. 2009. Mitochondrial DNA mutations and aging: devils in the details? Trends Genet. 25: 91-98. [Medline] [CrossRef]

15. Krupke, D.M., Begley, D.A., Sundberg, J.P., Bult, C.J., and Eppig, J.T. 2008. The Mouse Tumor Biology database. Nat. Rev. Cancer 8: 459-465. [Medline] [CrossRef]

16. Kujoth, G.C., Hiona, A., Pugh, T.D., Someya, S., Panzer, K., Wohlgemuth, S.E., Hofer, T., Seo, A.Y., Sullivan, R., Jobling, W.A., Morrow, J.D., Van Remmen, H., Sedivy, J.M., Yamasoba, T., Tanokura, M., Weindruch, R., Leeuwenburgh, C., and Prolla, T.A. 2005. Mitochondrial DNA mutations, oxidative stress, and apoptosis in mammalian aging. Science 309: 481-484. [Medline] [CrossRef]

17. Mito, T., Ishizaki, H., Suzuki, M., Morishima, H., Ota, A., Ishikawa, K., Nakada, K., Maeno, A., Shiroishi, T., and Hayashi, J.I. 2015. Transmitochondrial mito-mice $\Delta$ and mtDNA mutator mice, but not aged mice, share the same spectrum of musculoskeletal disorders. Biochem. Biophys. Res. Commun. 456: 933-937. [Medline] [CrossRef]

18. Mito, T., Kikkawa, Y., Shimizu, A., Hashizume, O., Katada, S., Imanishi, H., Ota, A., Kato, Y., Nakada, K., and Hayashi, J.I. 2013. Mitochondrial DNA mutations in mutator mice confer respiration defects and B-cell lymphoma development. PLoS One 8: e55789. [Medline] [CrossRef]

19. Nakada, K., Inoue, K., Ono, T., Isobe, K., Ogura, A., Goto, Y.I., Nonaka, I., and Hayashi, J.I. 2001. Inter-mitochondrial complementation: Mitochondria-specific system preventing mice from expression of disease phenotypes by mutant mtDNA. Nat. Med. 7: 934-940. [Medline] [CrossRef]

20. Roghani, T., Zavieh, M.K., Manshadi, F.D., King, N., and Katzman, W. 2017. Age-related hyperkyphosis: update of its potential causes and clinical impacts-narrative review. Aging Clin. Exp. Res. 29: 567-577. [Medline] [CrossRef]

21. Sato, A., Kono, T., Nakada, K., Ishikawa, K., Inoue, S., Yonekawa, H., and Hayashi, J.I. 2005. Gene therapy for progeny of mito-mice carrying pathogenic mtDNA by nuclear transplantation. Proc. Natl. Acad. Sci. USA 102: 1676516770. [Medline] [CrossRef]

22. Takai, D., Inoue, K., Shisa, H., Kagawa, Y., and Hayashi, J.I. 1995. Age-associated changes of mitochondrial translation and respiratory function in mouse brain. Biochem. Biophys. Res. Commun. 217: 668-674. [Medline] [CrossRef]

23. Taylor, R.W. and Turnbull, D.M. 2005. Mitochondrial DNA mutations in human disease. Nat. Rev. Genet. 6: 389-402. [Medline] [CrossRef]

24. Trifunovic, A., Wredenberg, A., Falkenberg, M., Spelbrink, J.N., Rovio, A.T., Bruder, C.E., Bohlooly-Y, M., Gidlöf, S., Oldfors, A., Wibom, R., Törnell, J., Jacobs, H.T., and Larsson, N.G. 2004. Premature ageing in mice expressing defective mitochondrial DNA polymerase. Nature 429: 417-423. [Medline] [CrossRef]

25. Wallace, D.C. 1999. Mitochondrial diseases in man and mouse. Science 283: 1482-1488. [Medline] [CrossRef] 